\title{
APORTE DE LA ACTUALIZACIÓN EN LA PRÁCTICA PROFESIONAL DE ORIENTADORAS Y ORIENTADORES \\ UPDATE CONTRIBUTION OF PRACTICE IN VOCATIONAL GUIDANCE
}

\author{
SECCIÓN ESPECIAL \\ Volumen 15, Número 1 \\ Enero - Abril \\ pp. 1-21
}

Este número se publicó el $1^{\circ}$ de enero de 2015

DOI: dx.doi.org/10.15517/aie.v15i1.17634

Viria Ureña Salazar

Sonia Parrales Rodríguez

Revista indizada en REDALYC, $\underline{\text { SCIELO }}$

Revista distribuida en las bases de datos:

CATÁLOGO DE LATINDEX, IRESIE, CLASE, DIALNET, DOAJ, E-REVIST@S, SHERPA/ROMEO, QUALIS, MIAR

Revista registrada en los directorios:

ULRICH'S, REDIE, RINACE, OEI, MAESTROTECA, PREAL, CLACSO 


\title{
APORTE DE LA ACTUALIZACIÓN EN LA PRÁCTICA PROFESIONAL DE ORIENTADORAS Y ORIENTADORES \\ UPDATE CONTRIBUTION OF PRACTICE IN VOCATIONAL GUIDANCE
}

\author{
Viria Ureña Salazar ${ }^{1}$ \\ Sonia Parrales Rodríguez
}

\begin{abstract}
Resumen: Este documento presenta un análisis de las reflexiones escritas elaboradas por 95 profesionales en Orientación, provenientes de diferentes zonas del país y que laboran en su mayoría en escuelas y colegios. Ellos matricularon el curso de actualización Orientación Vocacional: desarrollo y conducta vocacional, impartido por la Escuela de Orientación y Educación Especial, Sección de Orientación, de la Universidad de Costa Rica, durante el 2012 y 2013. Estas reflexiones son producto de los aportes teóricos estudiados y su práctica profesional. Del análisis de la información emergieron las siguientes categorías: la importancia de la actualización, la reflexión de la propia elección vocacional y la de los estudiantes, los aportes teóricos para su práctica profesional, el reconocimiento de las diferencias individuales.
\end{abstract}

Palabras clave: ORIENTACION VOCACIONAL,DESARROLLO VOCACIONAL, ELECCION VOCACIONAL, ACTUALIZACION, COSTA RICA.

\begin{abstract}
This document presents reflections developed by 95 professionals enrolled in the Career Counseling course, they belong from different parts of Costa Rica and the great majority work in schools and high schools. The participants enrolled the course Vocational Guidance upgrade: vocational develop and behavior, taught by the Counseling Section, Universidad de Costa Rica, in 2012 and 2013. Reflections were made about participants professional practice and the theoretical contributions impact of the course. The analysis categories were: Professional work, Uses of updating, Own career choice, Contributions of theoretical aspects, Recognition of individual differences, and Students process of career choice.
\end{abstract}

Key words: VOCATIONAL GUIDANCE, CAREER DEVELOPMENT, CAREER CHOICE, UPDATING, COSTA RICA

\footnotetext{
${ }^{1}$ Docente de la Escuela de Orientación y Educación Especial, Sección de Orientación, Universidad de Costa Rica. Dirección electrónica: viria.urena@ucr.ac.cr

${ }^{2}$ Docente de la Escuela de Orientación y Educación Especial, Sección de Orientación, Universidad de Costa Rica. Dirección electrónica: sonia.parrales@ucr.ac.cr
}

Documento recibido: 14 de julio, 2014

Enviado a corrección: 12 de agosto, 2014

Aprobado: 8 de diciembre, 2014

Este artículo tiene como objetivo compartir la experiencia de las autoras en el desarrollo del curso de actualización Orientación Vocacional: desarrollo y conducta 
vocacional, que ofrece la Escuela de Orientación y Educación Especial de la Universidad de Costa Rica; que se imparte desde el año 2012, Se retoman las reflexiones que realizan las personas participantes en torno a los aportes teóricos que les proporciona el curso para su práctica profesional.

Esta iniciativa de actualización nace de la necesidad identificada en una serie de consultas a las personas graduadas, como parte del proceso de autoevaluación de la carrera de Bachillerato y Licenciatura en Ciencias de la Educación con Énfasis en Orientación, con miras a la acreditación durante el 2011 y 2012.

En el informe que se presentó al Sistema Nacional de Acreditación de la Educación Superior (SINAES) se evidenció que, alrededor del $70 \%$ de las personas graduadas de la carrera opinaron que hay muy pocas o ninguna oportunidad de actualización profesional. Además, se señaló el desarrollo, la madurez vocacional y la Orientación vocacional ocupacional como temas que requieren ser fortalecidos en la práctica profesional (Escuela de Orientación y Educación Especial, 2011).

Por otro lado, es importante señalar que en el 2012 se aprobó en la Asamblea General Extraordinaria el Código de Ética del Colegio de Profesionales en Orientación. En el artículo 27 de este Código, se señala como competencia profesional, mostrar una conducta permanente de actualización. En el artículo 39 de docencia y actualización, se especifica que las personas profesionales en Orientación deben participar en programas de educación continua para garantizar la actualización de las competencias propias de la disciplina (Colegio de Profesionales en Orientación, 2012, pp. 8-9).

De esta manera, se plantea como proyecto de extensión docente el curso de actualización, Orientación Vocacional: desarrollo y conducta vocacional, con el objetivo de realimentar la intervención profesional de las personas graduadas de la carrera en el área vocacional. Para lograrlo, es necesario retomar aspectos teóricos, tales como el desarrollo vocacional, los constructos de conducta, madurez e identidad vocacional que permiten referirse a la elección vocacional desde una perspectiva de desarrollo y como una conducta en la cual inciden tanto factores psicogénicos como sociogénicos; para reflexionar sobre éstos y la práctica profesional en las instituciones educativas de quienes participan en el curso.

\section{Breve Referente Teórico}


A continuación se hace referencia a los aspectos teóricos que se desarrollan en el curso, los cuales constituyen el referente teórico de la información que se presenta en este artículo.

\subsection{Orientación Vocacional}

De acuerdo con Rodríguez (1998), la Orientación vocacional se concibe como un proceso que tiene lugar a lo largo de la vida de la persona, y que está inmersa en contextos diferentes a saber educativos, organizacionales, laborales y recreativos.

Por otra parte, Rivas-Martínez (2003) la define como el estudio de la conducta y el desarrollo vocacional a partir del cual se facilitan las elecciones en las personas, que le permitirán acceder al mundo laboral, así como el cumplimiento de las tareas propias de este desarrollo.

A partir de lo anterior, se evidencia que la Orientación vocacional supone un proceso de ayuda, dirigido a todas las personas, de acuerdo a la etapa del desarrollo en que se encuentran, desde una perspectiva de desarrollo humano y donde la vinculación al mundo del trabajo es siempre el referente. Reviste especial importancia las elecciones vocacionales que deben hacer las personas desde esta perspectiva.

\subsection{La elección vocacional como conducta desde una perspectiva del desarrollo humano}

La elección de una carrera u ocupación es el resultado de un proceso que se da a lo largo del desarrollo vocacional de las personas y en el cual influyen tanto factores psicogénicos como sociogénicos (Rivas-Martínez, 1995).

Esta decisión supone que en el paso de las personas por las diferentes etapas de desarrollo, con sus tareas y características propias, va configurando la conducta vocacional, en este caso, la elección vocacional.

La elección de una carrera u ocupación nace del análisis de la información que las personas tienen sobre sí mismas y del contexto en que se desenvuelve, e implica una escogencia entre varias alternativas educativas y laborales (Arguedas, Calderón, Cerdas, Jiménez y Vargas, 2006).

Lo anterior reviste especial importancia para la Orientación vocacional, porque permite a las y los profesionales de esta disciplina planificar su intervención; con el objetivo de facilitar el desarrollo vocacional de las personas orientadas y las elecciones implícitas en el 
proceso de su incorporación al mundo del trabajo, lo cual es requerimiento para la vida adulta.

\subsection{Madurez vocacional}

Concebir la elección vocacional desde la perspectiva del desarrollo permite referirse al constructo de madurez vocacional. De acuerdo a Super (1957) una persona puede ser considerada madura vocacionalmente si se encuentra cumpliendo las tareas, y se evidencian las características propias de la etapa del desarrollo en que se encuentran.

Para poder operacionalizar el constructo madurez vocacional, Super (1957) diseñó el Inventario de Desarrollo de Carrera (CDI), basado en diferentes escalas que permiten medir dos dimensiones fundamentales: la actitudinal y la cognitiva.

El Inventario consta de dos escalas actitudinales: la de Planificación de Carrera, y la de Exploración de Carrera; y de tres escalas cognitivas: Toma de Decisiones, Información del Mundo del trabajo y Conocimiento de la Ocupación Preferida.

Una propuesta alternativa a este modelo es la que presenta Crites (1978), al elaborar el Inventario de Madurez de Carrera (CMI). Este cuestionario incluye dos escalas que permiten analizar la actitud y las competencias que posee la persona. La Escala de Competencias analiza la Autoapreciación, la Información Ocupacional, la Selección de Metas y la Solución de Problemas; mientras que la Escala Actitudinal, analiza la Decisividad, la Implicación, la Independencia y el Compromiso de la persona ante el proceso de elección de carrera u ocupación.

Ambos inventarios coinciden en la clasificación de una persona madura vocacionalmente, si tiene una actitud favorable hacia los procesos de toma de decisiones, competencias y conocimientos para llevarlos a cabo; aunque esta coincidencia no se produce a la hora de matizar el tipo de actitudes y el tipo de competencias.

A partir de este constructo pueden surgir diferentes propuestas de programas de intervención para favorecer el desarrollo vocacional y la madurez de las personas orientadas.

\subsection{Identidad vocacional}

De acuerdo con Rocabert (2008) el constructo de madurez vocacional presenta dos limitaciones. Por una parte la dificultad de seguir manteniendo este concepto como un indicador propio y estable en la persona y como un rasgo permanente; y por otra parte la dificultad de mantener la estabilidad de los referentes respecto a lo que se considera como 
una persona madura vocacionalmente y la que no lo es, surgiendo un cuestionamiento en relación con las tareas propias a afrontar.

De esta manera, surge el concepto de identidad vocacional, que si bien es cierto no es novedoso, puede estar muy relacionado con la madurez vocacional; además de explicar el ¿por qué? una persona tiene una actitud y competencias favorables para tomar decisiones vocacionales efectivas y realistas, y ¿por qué? otras no.

Rocabert (2008) y su grupo de investigación retoman los aportes de Zimmermann y Bell (2002), Berzonsky, (2003) y Marcia (1966, 1980). El primero de acuerdo a la autora define la identidad vocacional como un proceso de revisión y replanteamiento de las metas de la vida, así como el grado de compromiso que la persona asume para el logro de éstas. Por su parte, Berzonsky (2003) considera que la identidad tiene un marcado carácter constructivo y de actividad, pues afirma que sirve para interpretar las propias experiencias y reestructurar el significado, propósito y dirección de la vida y Marcia $(1966,1980)$ se refiere a que la identidad constituye una organización interna autoconstruida de habilidades, creencias e historia individual que condiciona sus actuaciones, cuyos elementos están siendo agregados y desechados continuamente; cambiando la organización dinámica con el tiempo,

Propone además Rocabert (2008) los estatus de identidad que permiten operacionalizar el constructo de identidad a partir de que el estatus de identidad de una persona se define en función de dos dimensiones: la presencia versus ausencia de exploración de la identidad, entendida como la reflexión consciente de metas, alternativas, roles y valores; y la presencia versus ausencia de compromisos, que hace referencia a las convicciones fuertemente afianzadas relacionadas con la propia identidad.

El compromiso es definido como el grado de implicación, que la persona asume y manifiesta. La exploración es un proceso que refleja un examen genuino y la experimentación de direcciones alternativas y creencias, Marcia (1966, 1980), citado por Rocabert (2008).

Es sobre estas dos dimensiones, que Marcia (1980) citado por Rocabert (2008) postula los siguientes cuatro estatus de identidad:

a) Identidad difusa: que se caracteriza por la falta de compromiso y la exploración de opciones vocacionales. Se evidencia apatía y carencia de interés acerca de la dirección presente y futura de su vida; 
b) Identidad hipotecada: la persona no realiza una exploración activa de opciones, adopta compromisos y metas normativas parentales o significativas, convirtiéndola en conformista, obediente, con mentalidad cerrada y rígida;

c) Identidad moratoria: en la cual la persona asume una autonomía proactiva, de consideración de alternativas de identidad, pero con posibles niveles elevados de ansiedad e incertidumbre dada su condición en un periodo de transición psicosocial y

d) Identidad lograda: la persona evidencia que está en proceso hacia la madurez e inicio de la adultez; esto implica haber desarrollado un proceso de exploración, análisis e interiorización de alternativas de identidad (metas, roles y valores); y se formula firmes compromisos personales.

En este sentido, la propuesta de Berzonsky (2003) citado por Rocabert (2008) establece una relación entre los estatus de identidad y los estilos de procesamiento de la información. Este autor propone tres estilos: de evitación relacionado con la identidad difusa, el informacional relacionado con la identidad lograda y la moratoria, y el estilo normativo relacionado con la identidad hipotecada.

\section{La formación de profesionales en Orientación en el área vocacional, en la Universidad de Costa Rica}

Además del referente teórico, se considera necesario hacer una contextualización de la formación de profesionales de Orientación en la Universidad de Costa Rica.

A inicios del año 1964, el Ministerio de Educación Pública solicitó a la Facultad de Educación dar respuesta a la Reforma de la Segunda Enseñanza, con un plan de emergencia de seis semanas para formar a educadoras y educadores que atendieran el servicio de Orientación.

En la actualidad, en relación con el área vocacional de la Orientación, el Plan de estudios del Bachillerato y Licenciatura en Educación con Énfasis en Orientación, forma profesionales capaces de planear, ejecutar y evaluar programas y proyectos tendientes a favorecer el desarrollo vocacional de las personas, de acuerdo con la etapa del desarrollo en que se encuentran, para la vinculación al mundo del trabajo (Escuela de Orientación y Educación Especial, 2000).

Para lograr lo anterior, en el Plan de estudios de la carrera de Orientación vigente al 2011, en el grado de bachillerato se incluía el curso OE-2024 Orientación Vocacional; en 
este se abordaba principalmente aspectos teóricos a partir de los enfoques que permiten conocer el cómo las personas eligen una carrera u ocupación.

A partir del año 2013 se concreta un cambio en el Plan de estudios de bachillerato, en el que se incluyen dos cursos: el OE-2027 Orientación Vocacional I y el OE-2028 Orientación Vocacional II, en lugar de uno solo. En ambos programas se comprende el estudio de las diversas teorías y enfoques relacionados con el desarrollo y la elección vocacional; su aplicación en procesos de orientación individual y grupal y en estrategias que tiendan a favorecer el desarrollo vocacional, de la niñez y la adolescencia.

Como se observa, en el bachillerato se retoman los aspectos relacionados con lo que sucede antes de la elección de una carrera u ocupación. Por su parte, en la licenciatura se incluyen dos cursos, el curso OE-2052 Orientación Vocacional Ocupacional I, que es obligatorio para el estudiantado y como su nombre lo sugiere, profundiza en los contenidos que aborda a las personas en el mundo del trabajo; y el OE-2058 Orientación Vocacional II, que constituye la práctica profesional en esta área, como un curso optativo.

En estos cursos se enfatizan los enfoques evolutivos, por cuanto permite concebir que la elección que hacen las personas de una carrera u ocupación responde a un proceso, que se organiza en etapas y dentro de las cuales se pueden señalar tareas del desarrollo y características; de manera se puede hacer alusión al desarrollo vocacional de las personas a lo largo del ciclo vital.

Lo anterior permite a la Orientación vocacional, por su carácter educativo, anticipar su intervención, para favorecer el cumplimiento de las tareas propias del desarrollo y con ello el desarrollo vocacional; con miras a que las elecciones que hagan las personas en relación con lo vocacional, sean las más acertadas a partir su realidad personal y contextual.

Ya en el mundo del trabajo y desde la perspectiva del desarrollo profesional, entendido como el proceso a lo largo del cual la persona trabajadora calificada, enfrenta retos en relación con las demandas del mundo laboral y a sus competencias tanto personales como profesionales, los cuales pueden ser resueltos de manera más efectiva mediante procesos formativos, Mata (2002), el grupo de profesionales de Orientación, en este caso, requieren de actividades de actualización y de formación que les permita atender las demandas del contexto de manera oportuna y permanente, de ahí que desde la Universidad de Costa Rica se busca dar respuesta a través de la Extensión Docente, definida como 
el proceso mediante el cual la capacidad académica de la Universidad se traslada a la comunidad externa en actividades educativas, de difusión de conocimientos, actualización y capacitación de recursos humanos y servicios especiales de asesoría o laboratorio, con el propósito de colaborar con el desarrollo de grupos específicos y del país en general. (VAS, 2014, párr. 1)

No obstante se requiere por parte del y la profesional una actitud permanente para hacer la lectura de las demandas del contexto en el que laboran así como para el aprendizaje continuo y el desarrollo de las habilidades requeridas, que les permita dar respuesta a esas demandas, dentro de las que se encuentran, las que tienen que ver con la Orientación vocacional.

\section{Aspectos metodológicos}

Este artículo surge como se indicó anteriormente de la experiencia del curso Actualización en Orientación Vocacional: desarrollo y conducta vocacional, inscrito en la Vicerrectoría de Acción Social de la Universidad de Costa Rica, como un proyecto de extensión docente. Se plantea como objetivos generales los siguientes, Actualizar a profesionales en Orientación en el área vocacional, específicamente en lo relacionado con la conducta y el desarrollo vocacional y Reflexionar sobre la práctica profesional en relación con la Orientación vocacional.

Se desarrolla como un curso de aprovechamiento, el cual se organiza en cinco sesiones de trabajo, de ocho horas cada una. Del total de 40 horas, 32 son presenciales en el aula y 8 no presenciales, que se asignan para la elaboración de los trabajos del curso, que buscan facilitar la reflexión sobre la práctica profesional a través de la realización de ensayos.

En las sesiones presenciales los contenidos se desarrollan como clases magistrales, análisis y discusión de lecturas, así como a partir de la experiencia de las personas que participan. También, se cuenta con el apoyo de personas expertas para el desarrollo de los contenidos relacionados con el contexto, tales como el comportamiento del mercado laboral costarricense.

En las sesiones no presenciales se espera que cada participante realice una reflexión sobre los contenidos que se han desarrollado y su experiencia profesional, mediante la elaboración de síntesis y reflexiones a partir de la teoría. 
El contenido del curso ya definido de antemano parte de los aportes de los enfoques teóricos evolutivos de la Orientación Vocacional. Sin embargo, durante el desarrollo del curso se logran incluir algunos contenidos para dar respuesta a las necesidades específicas de cada grupo.

A continuación se presenta el contenido de cada una de las sesiones:

Sesión 1: Agrupación de enfoques teóricos, el enfoque evolutivo, elección y desarrollo vocacional, madurez vocacional, conducta Vocacional: aspectos psicogénicos y sociogénicos que inciden en ésta, e Identidad Vocacional

Sesión 2: Enfoque de toma de decisiones y su importancia, teoría de la casualidad, teoría de la incertidumbre positiva. Se realiza la primera reflexión, Contextualización de los aspectos teóricos estudiados en relación con la población orientada.

Sesión 3: La exploración vocacional, conducta exploratoria y autoeficacia vocacional.

Sesión 4: El uso de los instrumentos de exploración vocacional, validación y confiabilidad de los instrumentos de exploración vocacional, el Código de ética del CPO y las pruebas de exploración vocacional. Se elabora la segunda reflexión, Aportes Teóricos y Práctica Profesional.

Sesión 5: Presentación de una experiencia práctica y análisis del contexto nacional en relación con la elección de carrera: retos del y la profesional en Orientación.

Con el fin de valorar el logro de los objetivos, como se señalo anteriormente, se le pidió a las personas participantes, entre otras tareas, la elaboración de dos reflexiones en forma escrita. Una de ellas tiene que ver con el aporte de los conceptos teóricos retomados en el curso y la práctica profesional. Para rescatar la información aportada en los documentos que registran las reflexiones que se presentan en este artículo, se seleccionó el enfoque cualitativo que permite dar valor a todas las perspectivas y comprender a las personas dentro de su marco contextual (Taylor y Bogdan, 1996).

En este sentido, el enfoque cualitativo permite estudiar la realidad de los contextos naturales como la vida diaria, la interacción social; implica un componente objetivo (contexto natural) y otro subjetivo (significados atribuidos por los actores) el comportamiento, las manifestaciones, entre otros, para generar conocimiento (Taylor y Bogdan, 1996).

\subsection{Participantes}

En los cuatro cursos impartidos en los años 2012-2013 participaron un total de 95 profesionales en Orientación, que laboraban en escuelas, colegios diurnos, colegios técnicos 
y universidades públicas. La mayoría son mujeres, entre 20 y 50 años de edad y mayoritariamente con el grado académico de Licenciatura en Orientación.

Estas orientadoras y orientadores laboran en diferentes zonas del país como Cartago, Limón, Pérez Zeledón, San José, San Carlos, Guápiles, San Ramón, Palmares, Naranjo, Puntarenas, Alajuela y Heredia entre otras.

\subsection{Procedimiento para el análisis de la información}

Se analizan las reflexiones elaboradas por cada profesional participante en los cursos, con el objetivo de hacer referencia a los aportes teóricos retomados en las sesiones presenciales en relación con la práctica profesional.

Para el procesamiento y el análisis de las reflexiones de las y los participantes se utiliza el diseño de Taylor y Bodgan (1996), el cual consta de tres fases:

Primera fase, Descubrimiento en progreso: es un proceso que va muy ligado a la recolección de la información, ya que busca identificar temas emergentes, conceptos y proporciones que den sentido a la información que fue apareciendo para elaborar las categorías de análisis.

Segunda fase, Codificación: se produce cuando la información ya han sido recogida, incluye la codificación de la información y el refinamiento de la comprensión del tema en estudio. Incluye la reunión y análisis de todos los datos que se refieren a temas, ideas, conceptos, interpretaciones y proposiciones. Durante esta etapa de análisis, lo que inicialmente fueron ideas e intuiciones vagas se refinan, expanden, descartan o se desarrollan por completo. Una vez codificado los escritos, se elaboran las categorías de análisis, las cuales pueden surgir de los temas emergentes de los datos obtenidos y guiadas por los objetivos del proyecto.

De esta manera, se identificó en los documentos analizados seis categorías que se detallan a continuación:

a) Reflexionando sobre el quehacer profesional: se incluyen aquellos aspectos indicados por las personas participantes relacionados con la oportunidad que ofreció el curso para reflexionar respecto a la calidad y cantidad de acciones que están realizando en el área vocacional en las instituciones donde laboran.

b) Valorando la actualización: se refiere al reconocimiento que hacen las y los profesionales de Orientación participantes en el curso, sobre la importancia de la actualización 
c) Reflexionando sobre la propia elección vocacional: hace referencia a aquella información aportada por el grupo de profesionales de Orientación participante en el curso que reafirma o no su escogencia de la Orientación como profesión.

d) Reconociendo los aportes teóricos para la práctica profesional: en esta categoría se incluyeron los enunciados teóricos a los que hicieron referencia las personas participantes en los documentos que elaboraron.

e) Reconociendo las diferencias individuales: es el reconocimiento de las particularidades que tiene cada persona orientada con respecto a su elección vocacional y de manera consistente con la etapa de desarrollo que se encuentra en el momento del asesoramiento.

f) Sobre el proceso de elección vocacional del estudiantado: se refiere a las tareas que realizan las personas orientadas para la elección de carrera o laboral, como un continuo a lo largo de la vida.

Tercera fase, Relativización de los datos: en esta fase final se trata de relativizar los descubrimientos, es decir, de comprender los datos en el contexto en que fueron recogidos, ello implica el análisis y la interpretación de los mismos. Tiene que ver esta fase con los resultados que se presentan a continuación.

\section{Resultados}

A continuación se presentan los resultados según las seis categorías identificadas en la etapa de codificación.

a) Reflexionando sobre el quehacer profesional: De acuerdo a la información aportada por el grupo de profesionales de Orientación participante en el curso, en las reflexiones escritas los contenidos del curso les permitió reflexionar sobre su quehacer profesional, de manera que pudieron evaluar la labor realizada en las instituciones educativas, replantearse las acciones que están desarrollando así como las estrategias que están utilizando en los procesos de Orientación vocacional, plantearse nuevos retos que impliquen innovación y compartir y dar reconocimiento a todas y todos los profesionales por el trabajo con el estudiando en el área vocacional.

A continuación se detallan algunas manifestaciones que se incluyen en esta categoría y que dan evidencia de esta reflexión:

“...me reta a ser comprensiva e innovadora” G1 
“...este curso... es un llamado de atención constructiva, a mi labor profesional, lo cual me reta a mejorar aspectos propiamente vocacionales enfocados a toda la comunidad educativa en la cual laboro" G1

“... podemos implementar técnicas sin dejar de lado la creatividad y la innovación" G1

"Estimulación a los procesos de análisis y reflexión en cuanto al propio ejercicio profesional" G2

"Replantear las acciones que se implementan en las instituciones educativas... todo el aprendizaje enriquecerá nuestros planes anuales y demás actividades por realizar" G2

“... nos esforcemos por adquirir un compromiso real pensando en el bienestar integral de nuestros orientados... convicción de la importancia de nuestra labor, $y$ el trabajo en el desarrollo y fortalecimiento de todas aquellas habilidades $y$ características personales y profesionales que nos permitan desempeñarnos de la mejor manera en nuestro quehacer" G2

"Nuestro tiempo verdadero con la población... está marchitado, pero aun no ha desaparecido, y todavía hay entusiasmo y ganas de hacer las cosas que nos corresponden, aún se puede" G2

"Hacer un alto en el camino para meditar, revisar, cuestionar y re direccionar el quehacer profesional, es un ejercicio que pocas veces he realizado, pero darme esa oportunidad, ha sido el medio a través del cual la autoevaluación... me permite poner los pies en la tierra, recordarme que tengo mucho que aprender aún” G2

"Me parece muy importante el refrescamiento de temas, creo que... son muy importantes los temas vistos, muchos de ellos nos sirve en el trabajo diario" G3

“...refrescar conocimiento sobre la materia..."G3

"El curso actualización en Orientación Vocacional fue un proceso realmente recordatorio y renovación de conocimientos donde se reflexionó acerca de varios aspectos teóricos y de la práctica profesional" G4

"Es sorprendente observar las experiencias vocacionales de las y los compañeros del curso, donde se puede observar que cada uno realiza un trabajo excelente desde su contexto y de acuerdo a sus necesidades y sobre todo según las necesidades de la población orientada" G4 
"En general puedo decir que me siento satisfecha con el curso y más que la teoría que sí es muy importante me llevo las experiencias compartidas y nuevas estrategias prácticas de cómo complementar ambas partes en el mundo laboral como profesional en Orientación, profesión que cada día me convence más del impacto que puede generar en la sociedad" G4

"Permite una auto revisión para mejorar y dar lo mejor" G4

Como se observa los contenidos desarrollados en el curso permitió la reflexión por parte de las y los profesionales en Orientación, ya que logran mencionar tanto fortalezas como aspectos por mejorar en su trabajo, en el área vocacional. Lo anterior evidencia una actitud de autocritica constructiva y de aprendizaje, requisito indispensable para participar en actividades de actualización que favorezcan el desarrollo profesional de las personas trabajadoras, Mata (2002)

b) Valorando la actualización: el grupo de participantes reconoció la importancia de la actualización profesional por cuanto permite dar respuesta a los cambios del contexto y a las necesidades de la población orientada. También, reconocieron que se requiere conocer los cambios del mercado laboral para dar respuesta a las inquietudes que les plantea el estudiantado , para su elección vocacional.

Al respecto, expresaron lo siguiente:

“... resulta necesario que el o la orientadora esté capacitado y desarrolle habilidades para el manejo de técnicas y temáticas relacionadas a esta área, actualizando sus conocimientos constantemente, siendo un agente innovador $y$ creativo para atraer a sus estudiantes e incentivarlos a tomar las decisiones correctas para alcanzar la satisfacción personal y una vida plena” G1

"Es importante que se realicen más actualizaciones con mayor frecuencia, ya que al ser una profesión que está influenciada por diversos aspectos debemos estar en constante actualización” G2

"Este curso de actualización me ha brindado insumos para trabajar en orientación vocacional y me motiva a la actualización constante pero sobretodo a volver la mirada a la actualidad en mi región y promover este conocimiento en las personas involucradas en el proceso vocacional" G2 
“... partiendo de ésta realidad [constantes cambios en la economía, el medio ambiente, la ciencia, la tecnología, la política y la sociedad en general] surge la inquietud de que si nosotros como profesionales en orientación estamos conscientes de la necesidad de actualizarnos para poder contar con las herramientas que nos permitan afrontarnos a ésta época tan cambiante..." G2 "Es importante acotar que nuestros estudiantes cuentan con ciertos rasgos... y que las diferentes opciones que ofrece el mercado laboral requiere de diferentes tipos y configuraciones de estos rasgos..." G2

“Aplicación práctica, actualización teórica y metodológica” G4

"Demasiado útiles pues son un refrescamiento para mejorar como profesionales al actualizarse y revisarse" G4

"La importancia de conocer los movimientos del mercado laboral para las y los profesionales en Orientación, ya que es una información esencial para la toma de decisiones de las y los jóvenes" G4

Se evidencia en las frases anteriores que la actualización es valorada, ya que permite adquirir herramientas para enfrentar de una manera adecuada las demandas del entorno educativo y laboral, así como para hacer una valoración de las metodologías y estrategias utilizadas; igualmente de las actitudes que se tienen hacia el área vocacional en los procesos de Orientación que se implementan en las instituciones educativas. En este sentido, se reconoce que la actualización en el área vocacional contribuye en el ejercicio profesional que demanda el estudiantado en el contexto actual.

Es importante señalar que el hecho de participar en el curso, además de los aspectos señalados por el grupo profesional, evidencia una actitud ética, por cuanto se responde a lo indicado en el Código de Ética del Colegio de Profesionales en Orientación, en su artículo 27 , en el cual se señala como competencia profesional, el mostrar una conducta permanente de actualización y en el artículo 39, se especifica que deben participar en programas de educación continua para garantizar la actualización de las competencias propias de la Orientación (Colegio de Profesionales en Orientación, 2012).

c) Reflexionando sobre la propia elección vocacional: de acuerdo a la información aportada por las personas participantes en el curso se evidencia que este espacio de 
actualización les permitió reafirmar o replantearse la decisión de haber estudiado Orientación. Por ejemplo algunas y algunos participantes anotaron:

“...sobre la convicción de la importancia de nuestra labor. Si yo como orientadora estoy convencida de que mi trabajo, mis esfuerzos y dedicación son realmente importantes e influyentes en el proceso de formación de las personas, me sentiré comprometida con mi labor, y no permitiré que deje de lado lo que realmente debo hacer por otras actividades laborales que no me corresponden..." G2

“... estoy en un momento de sincero replanteamiento profesional... que se acrecentó mucho más durante estos días de capacitación” G2

"Considero que este curso... me deja preguntarme... porqué estudié orientación?" G2

“... ser profesional trasciende un título universitario..." G2

De acuerdo a lo expuesto, los contenidos que se desarrollaron en el curso a partir de lo indicado en las reflexiones, les permitió a las orientadoras y los orientadores reflexionar sobre su elección vocacional, como se indicó anteriormente. Lo anterior puede deberse a que cuando por ejemplo se abordan los aspectos psicogénicos y sociogénicos de la conducta vocacional, como la elección de una carrera, permite la reflexión de su propio proceso de toma de decisiones. En éste sentido recuérdese que la elección de una carrera es el resultado de un proceso que se da a lo largo del desarrollo vocacional de las personas, lo que supone el paso por las diferentes etapas del ciclo vital, con sus tareas y características, lo que va configurando la conducta vocacional entre ellas la elección vocacional, (Rivas- Martinez, 1995)

d) Reconociendo los aportes teóricos para la práctica profesional: En relación con esta categoría el grupo de participantes logró identificar los aspectos teóricos abordados en el curso, tales como, las etapas del desarrollo vocacional así como las de los procesos de Orientación Vocacional que desarrollan con sus estudiantes:

"El principio de irreversibilidad del desarrollo vocacional, donde los cambios o retrocesos se ven como experiencias de aprendizaje,... Otro aspecto que se puede retomar es que cuando se tiene vocación se realiza un trabajo excelente, ya que hay claridad en el proyecto de vida de la persona..." G4

"También se analizó algo importante dentro de la orientación vocacional lo cual es la exploración, de sí mismo y del ambiente, gran parte de la efectividad del 
proceso depende de la indagación, lo que se conoce como autoeficacia vocacional donde se relaciona la conducta, la cognición y el ambiente" G4

“... todos de gran valor... para trabajar con los estudiantes es la aplicación de técnicas llamativas, ya que considero que esta es una forma de involucrar a los jóvenes en la elección vocacional, siendo también responsabilidad de los orientadores el motivar a los y las estudiantes a una elección de proyecto de vida, y para ello necesitamos llamar la atención de nuestros jóvenes, ya que sin un rumbo claro las personas no toman decisiones acertadas y es donde se visualizan un sin número de personas insatisfechas" $G 1$

“...Lo ideal es encontrar el ajuste, la estabilidad y la autorrealización en el área vocacional y laboral... cuando esto no es posible $y$ no vivenciamos las gratificaciones que nos brinda tal selección, lo mejor es realizar un replanteamiento en búsqueda de la satisfacción profesional... en cualquier campo en que nos podamos desarrollar plenamente y disfrutando lo que hacemos" G3

"el desarrollo vocacional [es]... la interacción de factores psicológicos, económicos, sociales, físicos y de suerte, que moldean la carrera o secuencia de ocupaciones, trabajos y posiciones que una persona tiene durante el curso de la vida" G3

“... servir como medio de apoyo para guiar a las personas con la que trabajemos hacia el desarrollo y fortalecimiento de todas aquellas habilidades y adquisición significativa de conocimientos que les permitan adaptarse de forma proactiva al medio donde se desenvuelvan, con proyección hacia el logro de sus aspiraciones profesionales y laborales, en general, la búsqueda de la satisfacción en la construcción de su proyecto de vida" G2

"Mirar mi realidad circundante, en contraposición con la teoría, es un ejercicio que perdemos día a día, sabemos que lo hacemos, tiene un argumento o sustento teórico, pero que obviamos muchas veces..." G2

"Los elementos en cuanto a conducta vocacional que señala Rivas, se vieron evidenciados en aspectos como la importancia que para ellos tiene sentirse bien en lo que se hace (satisfacción y autorrealización)..." G2

"Es sumamente importante que nuestros orientados posean todo el conocimiento de los factores que inciden en la elección vocacional, tales como el conocimiento 
de sí mismo y el conocimiento de las diferentes oportunidades que ofrece el medio..." G2

Las afirmaciones anteriores representan como se indicó anteriormente un ejemplo de los aportes que el curso ofreció a las y los participantes en relación con los contenidos desarrollados y su práctica profesional, lo que evidencia el logro de los objetivos propuestos para el desarrollo del curso. Llama la atención a como se hace referencia a aspectos tales como los relacionados con los aspectos que influyen en la elección vocacional, al desarrollo vocacional y a la necesidad de favorecer el autoconocimiento y el contexto, lo que lleva a favorecer la conducta exploratoria. Por otro lado no se hace referencia a lo relacionado con la identidad vocacional, los aportes de la teoría de la incertidumbre positiva, de la casualidad y la de autoeficacia vocacional.

e) Reconociendo las diferencias individuales: El grupo de profesionales participante en el curso de actualización lograron reconocer las diferencias individuales que pueden darse en relación con el desarrollo vocacional y el contexto en que se desenvuelven las personas, por lo cual se deben hacer esfuerzos para contar con información específica en éste sentido “...nos permite comprender actitudes de los estudiantes en su proceso de desarrollo personal, académico y profesional. Es importante recordar las diferencias individuales que están presentes en todo este proceso de formación..." G1

“... debe existir un compromiso por conocer en la mayor medida de lo posible a nuestros estudiantes, su historia personal y familiar. Sólo de esta forma es mucho más probable que se logre un mayor impacto y mejores resultados en la aplicación de diferentes estrategias, las cuales deben responder a las necesidades reales de nuestros orientados" G2

“... debemos dedicarnos a conocer las características de los alumnos y el contexto en lo que estos se ven inmersos, conocer además sus capacidades 0 dificultades de aprendizaje para que la orientación sea dirigida hacia distintas áreas..." G2

“... tomar en cuenta en los estudiantes durante la preparación para una posible elección vocacional es el tipo de personalidad, que según Holland son fuertemente influenciados en el momento de realizar una escogencia, para ello 
es necesario que el estudiante sepa con claridad cuál tipo de personalidad tiene y con cuál ambiente se siente más cómo" G3

En relación con esta categoría es importante indicar que el grupo participante retoma conceptos que se desprenden del enfoque teórico evolutivo de la Orientación Vocacional, que es el que sustenta el desarrollo del curso y que como tal acoge los principios del desarrollo humano, entre ellos que la conducta vocacional se ve influenciada por factores psicogénicos y sociogénicos, lo que la hace particular en cada persona, (Rivas,1995).

f) Sobre el Proceso de elección vocacional del estudiantado: el grupo de profesionales reafirma en sus aportes que la elección vocacional es producto de un proceso a lo largo de la vida, que la Orientación vocacional no corresponde solamente a lo relativo a la elección de carrera y que implica una serie de acciones por parte de la persona orientada como el conocimiento de sí y de las oportunidades que le ofrece el medio. Lo anterior se evidencia cuando escribieron lo siguiente:

“... no es de ese tipo de decisiones que se toman todos los días, que no requieren de mucho tiempo para su análisis, son decisiones complejas que requieren de todo un proceso continuo a lo largo de los años..." G1

"La orientación vocacional permite que las personas conozcan sobre sus habilidades y capacidades, permitiéndole así, mejores herramientas a la hora de su elección vocacional" G1

"Se trata de que él y la estudiante se exploren así mismo, al medio que los rodea, sus cualidades y capacidades, que conozcan la realidad en la que se encuentran..." G1

“... trabajar con estas poblaciones desde tempranas edades [primaria] va a contribuir bastante en la elección vocacional, ofreciendo opciones... [para] su proyecto de vida" G2

“... a mi parecer se hace necesario que el desarrollo vocacional se inicie desde edades tempranas... logrando con ello que se intensifique y se mejore el desarrollo de la orientación y se dé un adecuado proceso vocacional" $G$ "[la orientación vocacional] ayuda a conocer cómo somos y cuáles son nuestros rasgos de personalidad, así como valorar la historia académica, familiar y social 
que cada uno ha vivido, nos ayudará a tomar una decisión académica acertada al elegir una profesión que sea compatible con nuestra situación personal real" G3

"El cómo tomar una decisión correcta, debe responder a una reflexión responsable de nuestros aspectos positivos y limitaciones... la información pertinente y veraz acerca del medio que nos rodea" G3

"[Hay que] lograr que los y las estudiantes reflexionen y analicen las consecuencias positivas y negativas que conlleva tomar una decisión" G3

"Prestar atención tanto al conocimiento de sí mismo como al mercado laboral, para tomar una decisión vocacional-ocupacional" G4

"Ver la Orientación Vocacional como algo continuo que no se centre solamente en el último nivel de secundaria" G4

Se observa como lo anotado por las y los profesionales de Orientación tiene correspondencia con los aportes del enfoque evolutivo, el cual constituye el referente teórico del curso, por cuanto permite referirse a la Orientación Vocacional como un proceso que se da a lo largo de la vida (Álvarez, 1995) donde los aspectos personales y contextuales van configurando la conducta vocacional (Rivas- Martínez, 1995).

Asimismo se logra reconocer por parte de las personas participantes en relación con los contenidos del curso y su práctica profesional acciones que reafirman su accionar así como aspectos en que deben mejorar.

\section{Conclusiones}

De acuerdo con el objetivo del presente artículo, se logra, a partir de la información aportada por el grupo profesional de Orientación que participó en el curso Orientación Vocacional: conducta y desarrollo vocacional, específicamente en la reflexión hecha sobre los aspectos teóricos abordados y su práctica profesional, agrupar en seis categorías los aspectos señalados por las personas participantes: reflexionando sobre el quehacer profesional, valorando la actualización, reflexionando sobre de la propia elección vocacional, reconociendo los aportes teóricos a la práctica profesional, reconociendo las diferencias individuales y lo sobre el proceso de elección vocacional del estudiantado, dentro de las cuales logran identificar aciertos que han tenido como profesionales de Orientación y así como aspectos por mejorar. 
Se evidencia también en la información aportada, supuestos teóricos propios de los aportes de los enfoques evolutivos para la Orientación vocacional, por ejemplo cuando hacen referencia al desarrollo vocacional y a la necesidad de concebir la Orientación vocacional como algo continúo y no como algo puntual en la etapa adolescente.

Además se reconoce la importancia de la actualización profesional como una manera de repasar aspectos teóricos y prácticos, así como para conocer aquellos más recientes, que complementan la formación inicial recibida como profesionales en Orientación.

Agrupar la información en las categorías anteriores y referirse a ellas permite afirmar que se logró cumplir con los objetivos propuestos del curso.

\section{Referencias}

Álvarez-González, Manuel. (1995). Orientación profesional. Barcelona: Cedecs.

Arguedas Brenes, Gustavo, Calderón Fernández, Esteban, Cerdas Blanco, Viviana, Jiménez Serrano, Elizabeth y Vargas Nieto, Ana Lidia. (2006). Manual para la enseñanza de la toma de decisiones a estudiantes de II Ciclo de la Educación General Básica (Tesis de licenciatura). Universidad de Costa Rica, San José, Costa Rica.

Colegio de Profesionales en Orientación. (2012). Código de Ética Profesional. San José, Costa Rica: Autor.

Crites, John. (1978). Career Maturity Inventory. Monterry, México: Mc Graw-Hill.

Escuela de Orientación y Educación Especial. (2011) Informe de Autoevaluación de la Carrera Bachillerato y Licenciatura de Educación con énfasis en Orientación. San José, Costa Rica: Autor.

Mata, Alejandrina (2002). La Orientación para el mejoramiento de la capacidad para el desempeño laboral del trabajador. Revista Actualidades en Orientación,7(1), 5-8.

Rivas-Martínez, Francisco. (2003). Psicología Vocacional: Enfoques del asesoramiento. Madrid, España: Editorial Síntesis.

Rivas-Martínez, Francisco. (1995). Manual de Asesoramiento y Orientación Vocacional. Madrid, España: Editorial Síntesis.

Rocabert Beut, Esperanza. (2008). Madurez e identidad vocacional. Conferencia llevada a cabo en la Escuela de Orientación y Educación Especial, Universidad de Costa Rica, San José, Costa Rica.

Rodríguez Moreno, María Luisa. (1998). Orientación educativa. Barcelona, España: Ceac.

Super, Donald. (1957). The Psychology of Careers. New York, Estados Unidos: Harper and Row. 
Taylor, Steve J. y Bogdan, Robert. (1996) Introducción a los Métodos Cualitativos de Investigación. Barcelona, España: Paidós Ibérica S. A.

Vicerrectoría de Acción Social. (2014). Acción social en la UCR. Recuperado de http://accionsocial.ucr.ac.cr/ed 